\title{
Effectiveness of Buspirone in Patients with Functional Dyspepsia: A Randomized, Double-Blind, Placebo-Controlled Study
}

\author{
Tarang Taghvaei ${ }^{1}$, Forouzan Elyasi ${ }^{2,3}{ }^{*}$, Zahra Rahbar $^{1}$, Farkhondeh Neyestani ${ }^{4}$
}

1. Gut and Liver Research Center, School of Medicine, Mazandaran University of Medical Sciences, Sari, Iran.

2. Sexual and reproductive health research center, Psychiatry and Behavioral Sciences Research Center, Addiction Institute, School of Medicine, Mazandaran University of Medical Sciences, Sari, Iran.

3. Department of Psychiatry, School of Medicine, Mazandaran University of Medical Sciences, Sari, Iran.

4. Diabetes Research Center, Mazandaran University of Medical Sciences, Sari, Iran.

* Corresponding Author:

Forouzan Elyasi, MD

Psychosomatic Ward, Imam Khomeini General Hospital, Razi Ave. Sari, Mazandaran, IR Iran.

Postal Code: 48157-33971.

Tel: +981133370885

Fax: +981133363754

Email: Forouzan.elyasi@gmail.com

Received: 09 Jun. 2021

Accepted: 02 Sep. 2021

\section{ABSTRACT}

\section{BACKGROUND}

Functional dyspepsia (FD) is a relatively common disorder whose pathogenesis has yet been poorly understood. There are still debates concerning definitions and the best possible treatments for this disorder. We aimed to assess the effectiveness of buspirone, a 5-hydroxytryptamine (HT) $)_{1 \mathrm{~A}}$ agonist, in improving the symptoms and quality of life $(\mathrm{QoL})$ as well as psychological dimensions in patients with FD.

\section{METHODS}

This study was a randomized, double-blinded, placebo-controlled trial performed on 30 patients with FD, residing in the city of Sari, northern Iran, from December 2017 to October 2018. Consecutive patients referring to a tertiary hospital with a clinical diagnosis of FD, according to the Rome IV criteria, were recruited. All patients were ethnically Persian and had normal upper endoscopy and negative histological evaluation results for any gastrointestinal disease or helicobacter pylori (H. pylori) infection while evaluating biopsy samples endoscopically. Exclusion criteria were being diagnosed with major psychiatric disorders, suicidal thoughts, recent treatments with psychoactive drugs, as well as major cognitive impairments. Patients were randomly assigned to receive either buspirone $(n=18)$ or placebo $(\mathrm{n}=12)$ for two months. The first group received buspirone $5 \mathrm{mg}$ three times a day for the first month and $10 \mathrm{mg}$ three times a day for the second month. During the treatment course, the patients were advised to report any adverse reactions. Also, both groups were evaluated by three questionnaires [demographic characteristics form, the 36-Item Short-Form Health Survey (SF-36), The Short-Form Leeds Dyspepsia Questionnaire (SF-LDQ) and Hospital Anxiety and Depression Scale(HADS)] the baseline and at the end of the 8th week by a blinded psychologist. Finally, data were analyzed using SPSS software (version 18). $P$ values $<0.05$ were considered statistically significant.

\section{RESULTS}

The most common symptoms of the patients were FD followed by heartburn. No significant differences were observed between buspirone and placebo groups regarding $\mathrm{QoL}(p=0.58)$, anxiety and depression ( $p=0.36$ ), and severity and frequency of FD symptoms $(p=0.22)$ before and after the intervention. In both groups, the overall QoL as well as HADS and SF-LDQ scores had significantly improved at the end of the study compared with the baseline.

\section{CONCLUSION}

Our findings indicate no significant effects associated with buspirone on the clinical course of FD, compared with placebo. More studies are needed to introduce effective therapies according to the pathophysiology of FD.

\section{KEYWORDS:}

Buspirone, Therapy, Clinical Trial, Dyspepsia

Please cite this paper as:

Taghvaei T, Elyasi F, Rahbar Z, Neyestani F. Effectiveness of Buspirone in Patients with Functional Dyspepsia: A Randomized, Double-Blind, Placebo-Controlled Study. Middle East J Dig Dis 2021;13: 302-313. doi: 10.34172/mejdd.2021.239 


\section{INTRODUCTION}

Dyspepsia is a common symptom; however, up to $75 \%$ of the affected patients have no underlying cause on diagnostic evaluation. They are known as having functional dyspepsia (FD). Based on the Rome III criteria, this condition is defined as the presence of one or more symptoms e.g. postprandial satiety, pain, or epigastric burning over the past three months or persisting symptoms for at least six months. The diagnosis is accordingly approved when there are no signs of other anatomical or structural diseases. ${ }^{1}$ Moreover, multiple pathophysiological mechanisms are involved in FD, including delayed gastric emptying, antral motility disorders, vagal neuropathy, postprandial gastric adjustment disorder, altered visceral sensation, post-infectious gastroenteritis, gastroduodenal inflammation, and psychological issues. Accordingly, drugs increasing gastrointestinal (GI) motility, stimulating adjustment of the gastric fundus, and reducing excessive visceral sensitivity to gastric distension are potential therapeutic options for FD. ${ }^{1}$ Psychological distress, in particular anxiety, has also been associated with this condition. In some patients, psychological conditions may develop before the onset of FD. In others, they occur after the onset of digestive symptoms. Moreover, central pain processing may be abnormal in individuals with FD; however, it is unclear whether this is a complication or an early sign of the disease. ${ }^{2}$

Given the potential role of the gut-brain axis (GBA) and the abnormal central pain processing in $\mathrm{FD}$, antidepressants have been so far proposed as viable treatments for this condition. Nevertheless, clinical trials regarding the effects of antidepressants in FD have been only conducted over the past decade. ${ }^{2}$ Also, selective serotonin reuptake inhibitors (SSRIs) and serotonin-norepinephrine reuptake inhibitors (SNRIs) have been proposed as acceptable alternative treatments for the management of other situations rather than depression. ${ }^{3}$ Thase and colleagues reported no beneficial effects by the use of venlafaxine for 8 weeks on 160 patients with FD. ${ }^{4}$ In another placebo-controlled trial on the impacts of sertraline on 193 patients with FD in China, 28\% of the patients assigned to either sertraline or placebo groups had experienced complete symptom-free periods..$^{5}$ Also, Ly and co-workers evaluated the effects of mirtazapine on 34 patients with FD, experiencing weight loss. Over 8 weeks, significant improvements had been observed in the quality of life $(\mathrm{QoL})$ in patients receiving mirtazapine compared with those assigned to the placebo. ${ }^{6}$ In a recent multicenter clinical trial in North America, 292 patients with FD were treated with amitriptyline, escitalopram, or placebo. After 10 weeks, the response rates were $53 \%, 38 \%$, and $40 \%$, respectively. These findings suggest that tricyclic antidepressants such as amitriptyline seem to be more effective than selective serotonin reuptake inhibitors or serotonin-norepinephrine drugs in the treatment of FD. ${ }^{2}$

Besides, serotonin 5-hydroxytryptamine (5-HT) is a neurotransmitter particularly involved in regulating GI tract motility, smooth muscle tone, mucosal secretion, and neural signaling. Accordingly, $95 \%$ of the total body serotonin is secreted by enterochromaffin cells of the digestive system. The physiological role of intestinal serotonin is executed by the activation of serotonin receptors expressed in the GI tract. These specific serotonin receptors are therapeutic targets of the drugs used for the treatment of motility-related digestive disorders. Seven distinct molecular subtypes of serotonin receptors have been thus far identified that differ in terms of functional and structural features. 5-HT (serotonin) receptor $1 \mathrm{~A}\left(5-\mathrm{HT}_{1 \mathrm{~A}}\right), 5$-hydroxytryptamine (serotonin) receptor $1 \mathrm{~B}\left(5-\mathrm{HT}_{1 \mathrm{~B}}\right), 5-\mathrm{HT}_{2 \mathrm{~A}}, 5-\mathrm{HT}_{2 \mathrm{~B}}, 5-\mathrm{HT}_{3}, 5-\mathrm{HT}_{4}$, and $5-\mathrm{HT}_{7}$ receptors are expressed in the nervous system and the smooth muscles of the GI tract. As well, 5- $\mathrm{HT}_{1 \mathrm{~A}}$ and 5- $\mathrm{HT}_{1 \mathrm{~B}}$ are inhibitors, and 5- $\mathrm{HT}_{4}$ and 5- $\mathrm{HT}_{3}$ are simulators for neurotransmitter release. The activation of the $5-\mathrm{HT}_{1 \mathrm{~A}}$ receptor also inhibits the release of acetylcholine following smooth muscle contraction. Buspirone is a non-selective agonist of $5-\mathrm{HT}_{1 \mathrm{~A}}$ receptor enhancing gastric function in patients with FD. Accordingly, buspirone decelerates gastric emptying in humans by increasing the lower esophageal sphincter tone. ${ }^{8}$

Controversial results have been so far obtained on the therapeutic effects of 5-HT $\mathrm{HA}_{1 \mathrm{~A}}$ agonists on FD in numerous studies. For example, Tabib and colleagues 8 indicated the superiority of 5-HT ${ }_{1 \mathrm{~A}}$ agonists in improving FD symptoms and QoL in these patients. On the other hand, Miwa and others ${ }^{9}$ demonstrated the higher efficacy of $5-\mathrm{HT}_{1 \mathrm{~A}}$ agonists on the improvement of FD symptoms; however, they observed no significant difference between $5-\mathrm{HT}_{1 \mathrm{~A}}$ agonists and placebo in improving QoL and psychological dimensions. Moreover, Tack and co-workers reported that 4-week buspirone administration improved the symptoms of delayed gastric emptying in patients with FD. ${ }^{10}$ Nevertheless, 4-week 
administration of R-137696 as another 5-HT $1 \mathrm{~A}$ agonist did not significantly recover the symptoms of excessive visceral sensitivity, gastric distension, and gastric adjustment compared with placebo in patients with FD. ${ }^{11}$

The clinical course of FD may follow normal, chronic, or oscillating patterns. The disease recurrence has also been reported following symptom-free periods in long-term follow-ups. About $15-20 \%$ of patients with FD experience persistent disease, while symptom-free periods have been observed in $50 \%$ of such patients. In the remaining patients (30-35\%), the symptoms follow a fluctuating pattern similar to other functional digestive disorders. ${ }^{2}$

The present study was developed because the number of randomized, double-blind, placebo-controlled clinical trials comparing buspirone with placebo in patients with FD has been thus far limited. With reference to other studies, it was assumed that buspirone could improve the QoL in patients with FD and reduce the symptoms of anxiety, depression, and pain. This randomized and double-blind trial accordingly examined the effect of buspirone on FD.

\section{MATERIALS AND METHODS}

The present study was a randomized, double-blind, placebo-controlled trial performed on patients with FD referring to the gastroenterology clinic at a tertiary hospital in the city of Sari, northern Iran, from December 2017 to October 2018. In this interventional study, the statistical sample included 40 outpatient FD patients who completed the Rome IV criteria for FD. Patients must have $\geq 1$ of the following symptoms for 3 months prior to the symptom onset $\geq 6$ months earlier: bothersome postprandial fullness, bothersome early satiation, bothersome epigastric pain, bothersome epigastric burning. There is no evidence of organic, systemic, metabolic, or structural disease likely to explain symptoms. ${ }^{12}$ Endoscopy was also carried out with biopsy taking in all patients to rule out any structural pathology, gastroesophageal reflux disease (GERD), and eosinophilic esophagitis (EoE). Written informed consent was obtained from all the participants before their recruitment in the study.

The inclusion criteria were adult patients with normal endoscopic findings and a history of dyspepsiarelated symptoms within the past 3 months, who were diagnosed as having FD. The exclusion criteria were the presence of psychiatric disorders including acute anxiety and depression, early diagnosis of psychotic disorders, active substance abuse or drug dependence, suicidal thoughts, history of eating disorders, psychotic or bipolar disorder, recent treatments with psychoactive drugs, and major cognitive impairments. Moreover, findings in upper GI endoscopy or esophagogastroduodenoscopy (EGD) such as ulcer, cancer, erosion, and GERD, history of gastric or esophageal surgeries, use of non-steroidal anti-inflammatory drugs (NSAIDs), gastric reflux, celiac disease, and acute helicobacter pylori (H. pylori) infection (diagnosed by the rapid urease test or histological evaluation of gastric biopsy samples) were considered as other exclusion criteria. In addition, unstable angina, hypertension, and asthma, history of hypersensitivity to buspirone, pregnancy, lactation, and inappropriate contraception were regarded as further exclusion criteria in this study. Accordingly, all potential participants underwent a medical evaluation for such exclusion criteria. The medical evaluation included taking a medical history, routine physical examination, blood biochemistry, and urine analysis for drug abuse, along with a pregnancy test if necessary. The minimum work-up to exclude organic diseases included taking medical history by interview, physical examination, an upper gastroduodenal endoscopy, blood investigation including thyroid-stimulating hormone (TSH), white blood cell count (WBC), and C-reactive protein (CRP), accompanied by abdominal ultrasonography.

This study was a single-center, randomized, placebo-controlled trial. The targeted accrual in the original protocol was 30 participants. Assuming 20\% attrition rate, having 40 participants would yield $90 \%$ power to make a difference in the QoL mean pre/post-changes between the groups at the significance level of 0.05. Therefore, the final accrual was 40 randomized participants.

Randomization and the release of the study medication (Buspirone or placebo) were performed by a clinical pharmacist. The pharmacist used block randomization, with blocks of 10 participants, which was blinded to the investigators until the end of the study. Buspirone and placebo were also packed in identical 
sealed boxes, marked with the specific study code and participant code. But they had the same shapes. Both investigators and patients were also blinded to the intervention.

The patients were randomly assigned to intervention (receiving buspirone) or control (taking placebo) groups ( $n=20$ per group). One group received a placebo (namely, starch), and the other group was given buspirone (Iran Daru Co. Iran). The intervention group received buspirone $5 \mathrm{mg}$ three times a day for the first month and $10 \mathrm{mg}$ three times a day for the second month. It should be noted that the dose could be modified at any time in terms of clinical efficacy and side effects. Both patients and the rater (FN) were blinded to group allocation. The gastroenterologist was also blinded to the groups. Besides, the person entering the patients into the groups was different from the person who scored the measurement tools. The side effects of the drug were further evaluated on a weekly basis by a physician through open-ended questions e.g. "Have you ever had any side effects since the last time we met? Such as feeling cold, flu, nausea, increased appetite, dry mouth, headache, or any other problems". Furthermore, an 8-week intervention period was chosen because of the delayed onset of the buspirone effect. ${ }^{13,14}$

Furthermore, three questionnaires (demographic characteristics form, the 36-Item Short-Form Health Survey (SF-36), The Short-Form Leeds Dyspepsia Questionnaire (SF-LDQ) and Hospital Anxiety and Depression Scale (HADS) were accordingly completed at the baseline and at the end of the 8th week by a blinded psychologist who was not informed of patients' assignments to either buspirone or placebo groups.

\section{Demographic Characteristics Form}

The patients' demographic characteristics including age, sex, height, weight, marital status, level of education, number of children (if any), place of residence, level of income, occupation, and clinical features such as a history of hypertension, thyroid problems, and other physical diseases, as well as menstrual status, regular exercise, depression, and familial history of
FD were recorded in a questionnaire.

\section{6-Item Short Form Survey (SF-36)}

The QoL of the patients was assessed using SF-36 questionnaire, comprised of 36 items and eight scales comprised of 2-10 items. ${ }^{15}$ The items were related to both personal characteristics (sex, age, marital status, occupation, etc.) and activities of daily living. The items in the second part were to review the patients' physical and mental capabilities in eight sections. Finally, the scores of the physical and the psychological components could be calculated. Each item was scored from zero to 100 , and then the overall mean could be calculated. The subscales of this questionnaire included physical functioning $(\mathrm{PF})$, role limitations due to physical health (RP), role limitations due to emotional problems (RE), energy/fatigue (EF), emotional well-being (EW), social functioning (SF), pain $(\mathrm{P})$, and general health $(\mathrm{GH})$. The mean scores in each of the eight subscales were also assigned from zero to 100. Accordingly, items no. 3, 4, 5, 6, 7, 8, 9 , 10,11 ; items no. $12,13,14,15$; items no. $16,17,18$; items no. 19, 23, 27, 29; items no. 24, 25, 26, 28, 31; items no. 30, 20; items no. 21, 22, 32; and finally items no. $1,33,34,35,36$, were respectively applied to assess the PF, RP, RE, EF, EW, SF, P, and GH subscales. The overall subscale of physical health was considered as the sum of the PF, RP, P, and GH subscales. In addition, the subscale of mental health was regarded as the sum of the RE, EF, EW, and SF subscales. ${ }^{15}$ It should be noted that the reliability and the validity of the Persian version of the SF-36 had already been approved by Montazeri and colleagues in $2005 .^{16}$

\section{Hospital Anxiety and Depression Scale (HADS)}

HADS presents a useful and brief screening tool for the symptoms of depression and anxiety in patients with physical problems. This questionnaire consists of two parts with 14 items, wherein anxiety and depression are evaluated by seven items. Each component of the scale is also scored from zero to 3 , giving a score range of 0-21 for depression and anxiety subscales. The validity and reliability of the Persian version of HADS had been previously confirmed by Kaviani and colleagues. ${ }^{17}$ 


\section{The Short-Form Leeds Dyspepsia Questionnaire (SF-LDQ)}

The SF-LDQ is a routine tool used for predicting the severity of FD symptoms. The SF-LDQ contained questions about indigestion frequency, heartburn frequency, regurgitation frequency, nausea frequency, indigestion severity, heartburn severity, regurgitation severity, and nausea severity. The highest achievable score in the questionnaire is 48. Moreover, scores $1-12,13-24,25-36$, and 37-48 represent mild, moderate, severe, and very severe conditions, respectively. ${ }^{18}$

\section{Statistical Analysis}

The data were analyzed using the SPSS software (version 18). All the variables were thus encoded. Mean, and standard deviation (SD) were also employed to report the continuous variables. The categorical variables were further stated as numbers (percentage). Kolmogorov-Simonov test was additionally utilized. Comparably, $t$ tests were used for comparing the continuous variables between both groups. Chisquare test and Fisher's exact test were applied to compare the distribution of the qualitative variables between both intervention and placebo groups. To compare the means of the quantitative variables between the two study groups, either independent samples t-test (for the normally distributed data) or MannWhitney U test (for the non-normally distributed data) were used. For pre/post-intervention comparisons, paired samples t-test (for the data with normal distribution) or Wilcoxon signed-rank test (the data with non-normal distribution) were utilized. $P$ values less than 0.05 were considered statistically significant.

\section{Ethical Considerations}

Before participating in the study, the objectives and design of the study were explained to each patient. Then, they were requested to sign an informed written consent prior to the study. These patients could also withdraw from the study if they were not willing to cooperate at any stage. Of note, all the patients' information was remained confidential throughout the study and afterward. This clinical trial was also approved by the Ethics Committee of Mazandaran University of Medical Sciences (MUMS), Sari, Iran. This trial was conducted based on the Declaration of Helsinki and subsequent revisions of the Helsinki treaty (ethics code: IR.MAZUMS.IMAMHOSPITAL.REC.1397.2940) (IRCT ID: IRCT20180405039198N1).

\section{RESULTS}

Out of the 40 patients with FD, three patients in the placebo group withdrew from the study. One of them did not refer to continue the treatment and did not answer his phone. Two patients in the placebo group also stopped the treatment after one month by complaining about the exacerbation of their symptoms. Finally, the data of 30 patients were used to accomplish the study (18 cases in the intervention and 12 individuals in the control groups) and to compare the QoL, psychological dimensions, and symptoms of FD between the study groups (figure 1).

Patients' Demographic and Clinical Characteristics

No significant difference was observed between both groups regarding clinical (i.e. age, height, weight, and body mass index (BMI), and demographic (namely, marital status, level of education, number of children, place of residence, level of income, and occupation, (table1) characteristics.

\section{QoL in Patients with FD using SF-36}

The QoL scores before and after 8 weeks in buspirone-receiving and placebo groups are indicated in table 2. Accordingly, there were no differences between both groups respectively at the baseline in terms of physical health subscale $(51.97 \pm 19.88$ vs. $63.43 \pm 14.46, p=0.09)$ and mental health subscale $(50.19 \pm 24.20$ vs. $39.98 \pm 19.09$, $p=0.23$ ), respectively. Also, no significant difference was found between the two groups regarding the physical health subscale $(68.40 \pm 15.53$ vs. $63.43 \pm 20.59, p=0.26)$ and mental health subscale $(69.49 \pm 20.31$ vs. $69.07 \pm 18.41$, $p=0.95)$ at the 8 th week, respectively.

\section{Anxiety and Depression in Patients with FD}

No significant differences were observed between both groups at the baseline or after 8-week intervention; however, the groups demonstrated significant improvements 


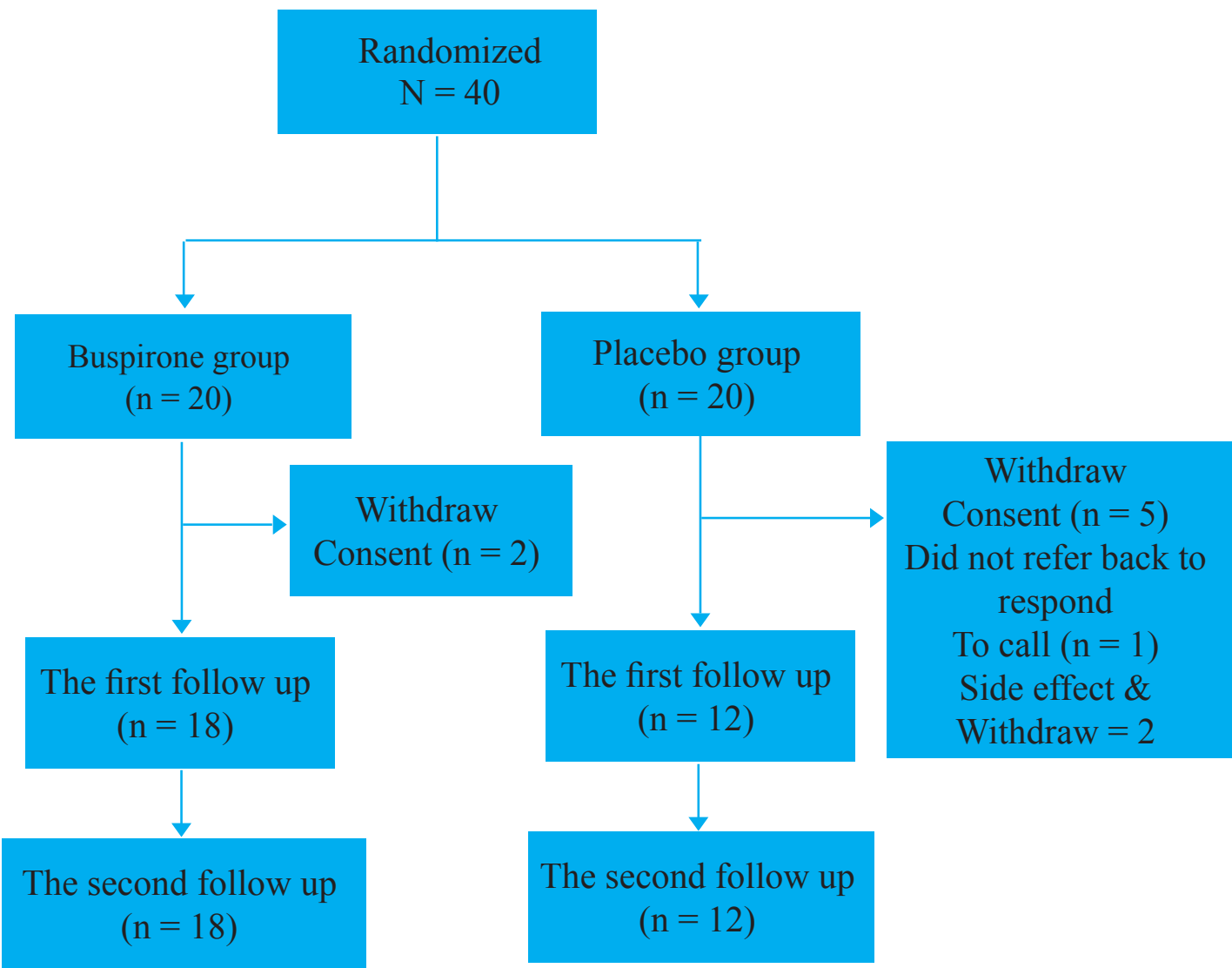

Fig. 1: Analyzed to compare quality of life, psychological dimensions, and symptoms of functional dyspepsia between the two study groups

in anxiety and depression at the end of the study (table 3).

\section{FD Symptoms using SF-LDQ}

The most common symptoms among the patients were FD followed by heartburn (table 3). Although no significant difference was reported between the two groups at the baseline or at the end of the intervention, both groups showed improvements in general symptoms of dyspepsia at the end of the study compared with the baseline (table 4).

\section{Side Effects}

No serious side effects were found. The most common side effects in the buspirone group were increased appetite (15\%), drowsiness (20\%), and fatigue (5\%). As well, the most common side effects in the placebo group were increased appetite (10\%) and dry mouth (10\%).

\section{DISCUSSION}

In this randomized clinical trial, we aimed to assess the effectiveness of buspirone, a 5- $\mathrm{HT}_{1 \mathrm{~A}}$ agonist, in improving FD symptoms as well as QoL and psychological dimensions in the affected patients. In this study, no significant difference was observed between the two groups. No significant differences were detected comparing anxiety and depression scores between the buspirone- and placebo-receiving groups. Overall, significant improvements were observed in the total scores of QoL and its five specific related subscales (i.e. RP, RE, EF, EW, and $\mathrm{P})$, as well as the two general subscales (i.e. physical and mental health) in patients taking buspirone for 8 weeks. In addition, significant improvements were observed in post-intervention HADS and SF-LDQ scores in buspirone group. In the placebo group, the patients demonstrated a significant improvement in the total score of SF-36 and general mental health subscale (namely, energy/fatigue, emotional well-being, and physical functioning) compared with the baseline status. Unlike the buspirone group, the placebo group showed no improvements 
Table 1: Clinical and demographic characteristics of patients with $\mathrm{FD}^{*}$ in buspirone and placebo groups

\begin{tabular}{|c|c|c|c|c|}
\hline Characteristics & & $\begin{array}{c}\text { Buspirone group } \\
\mathrm{N}=\mathbf{1 8} \\
\mathrm{N}(\%)\end{array}$ & $\begin{array}{c}\text { Placebo group } \\
\mathbf{N}=12 \\
\mathbf{N}(\%)\end{array}$ & $p$-valuc \\
\hline \multirow{4}{*}{ Marital status } & Single & $0(0)$ & $3(25)$ & \multirow{4}{*}{0.06} \\
\hline & Married & $17(94.4)$ & $9(75)$ & \\
\hline & Widowed & $0(0)$ & $0(0)$ & \\
\hline & Divorced & $1(5.6)$ & $0(0)$ & \\
\hline \multirow{7}{*}{ Level of education } & Illiterate & $0(0)$ & $0(0)$ & \multirow{7}{*}{0.55} \\
\hline & Primary school 1 & $3(16.67)$ & $0(0)$ & \\
\hline & Primary school 2 & $2(11.11)$ & $3(25)$ & \\
\hline & Diploma & $6(33.33)$ & $3(25)$ & \\
\hline & High school & $2(11.11)$ & $1(8.3)$ & \\
\hline & Bachelor's degree & $4(22.22)$ & $3(25)$ & \\
\hline & $\begin{array}{l}\text { Master's or PhD } \\
\text { degree }\end{array}$ & $1(5.6)$ & $2(16.7)$ & \\
\hline \multirow{2}{*}{ Having children } & Yes & $14(77.78)$ & $7(58.3)$ & \multirow{2}{*}{0.41} \\
\hline & No & $4(22.22)$ & $5(41.7)$ & \\
\hline \multirow{2}{*}{ Place of residence } & Urban & $13(72.22)$ & $10(83.3)$ & \multirow{2}{*}{0.66} \\
\hline & Rural & $5(27.78)$ & $2(16.7)$ & \\
\hline \multirow{3}{*}{ Housing } & Householder & $16(88.89)$ & $10(83.34)$ & \multirow{3}{*}{0.45} \\
\hline & Tenant & $0(0)$ & $1(8.33)$ & \\
\hline & unknown & $2(11.11)$ & $1(8.33)$ & \\
\hline \multirow{3}{*}{$\begin{array}{l}\text { Level of income } \\
\text { (Rials) }\end{array}$} & $\begin{array}{l}5000000- \\
10000000 \\
\end{array}$ & $2(11.11)$ & $0(0)$ & \multirow{3}{*}{0.43} \\
\hline & $>10000000$ & $12(66.67)$ & $8(66.67)$ & \\
\hline & Not reported & $4(22.22)$ & $4(33.33)$ & \\
\hline \multirow{4}{*}{ Occupation } & Part-time & $1(5.6)$ & $1(8.3)$ & \multirow{4}{*}{0.30} \\
\hline & Full-time & $4(22.22)$ & $5(41.7)$ & \\
\hline & Housewife & $13(72.22)$ & $5(41.7)$ & \\
\hline & Student & $0(0)$ & $1(8.3)$ & \\
\hline \multirow{2}{*}{ Regular exercise } & Yes & $3(16.67)$ & $1(8.3)$ & \multirow{2}{*}{0.53} \\
\hline & No & $15(83.33)$ & $11(91.67)$ & \\
\hline \multirow{2}{*}{$\begin{array}{l}\text { Familial history of } \\
\text { FD }\end{array}$} & Yes & $2(11.11)$ & $5(41.7)$ & \multirow[t]{2}{*}{0.08} \\
\hline & No & $16(88.89)$ & $7(57.3)$ & \\
\hline \multirow{2}{*}{$\begin{array}{l}\text { History of referring to } \\
\text { psychiatrists }\end{array}$} & Yes & $7(38.89)$ & $1(8.3)$ & \multirow{2}{*}{0.09} \\
\hline & No & $11(61.11)$ & $11(91.67)$ & \\
\hline
\end{tabular}

* FD: Functional Dyspepsia

in the overall physical health subscales and its related items (physical functioning, general health, and pain) compared with the baseline. However, HADS and SFLDQ scores significantly improved in the placebo group at the end of the study as compared with the baseline.

Controversial results have been so far provided on the therapeutic effects of 5-HT $1 \mathrm{~A}$ agonists on FD in different studies. For example, Miwa and colleagues ${ }^{9}$ and Tabib and co-workers ${ }^{8}$ reported improvements in the QoL and psychosocial dimensions in patients with FD in both groups of patients receiving $5 \mathrm{HT}_{1 \mathrm{~A}}$ agonist and placebo. In a multicenter double-blinded placebo-controlled trial in 2009 by Miwa and colleagues, the impacts of 4-week administration of tandospirone citrate, a $5-\mathrm{HT}_{1 \mathrm{~A}}$ receptor agonist, was evaluated on the QoL (assessed by SF-8) and psychological dimensions (measured by the State- 


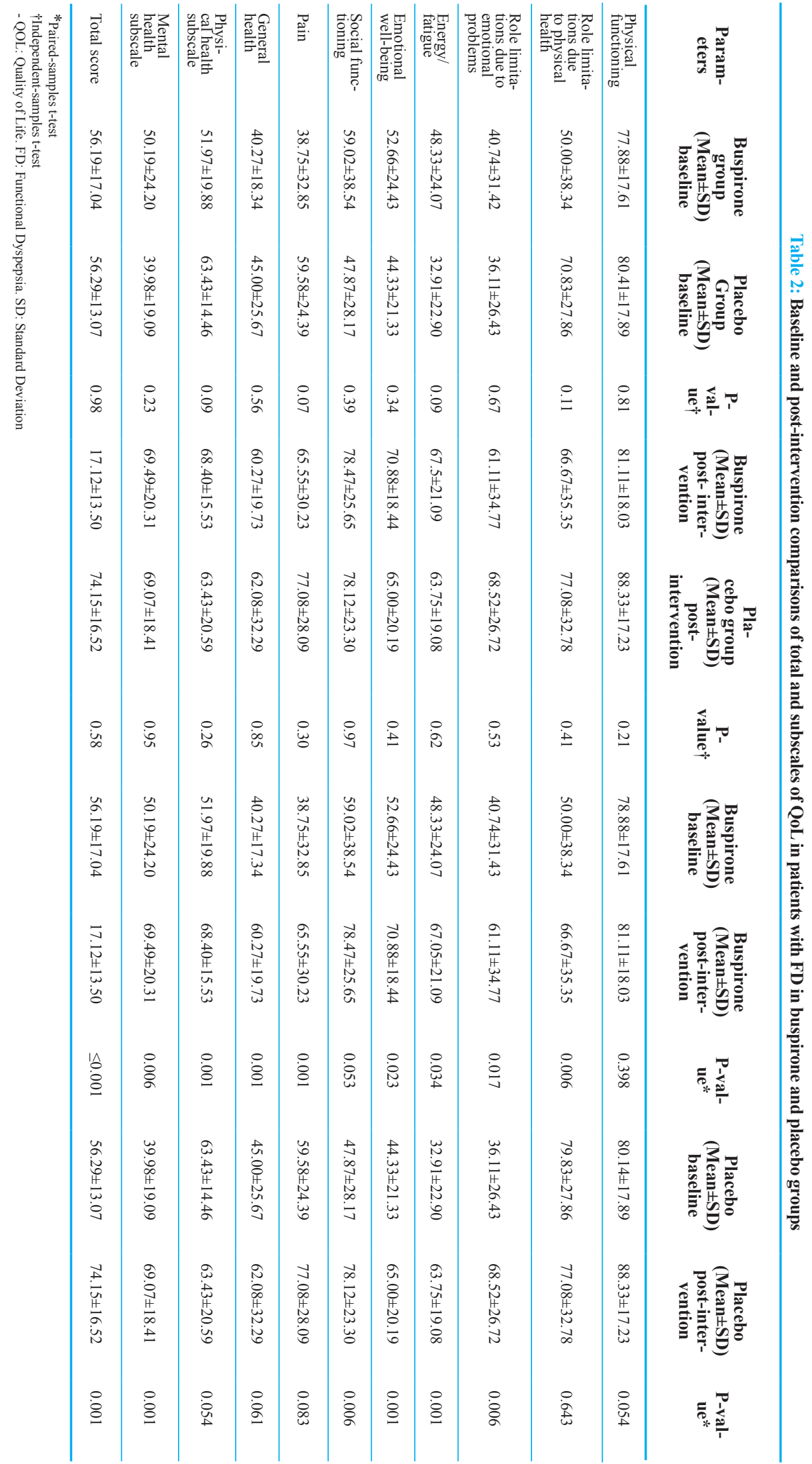


Table 3: Comparison of total subscale scores of HADS and LDQ-SF between placebo and buspirone groups at baseline and post-intervention

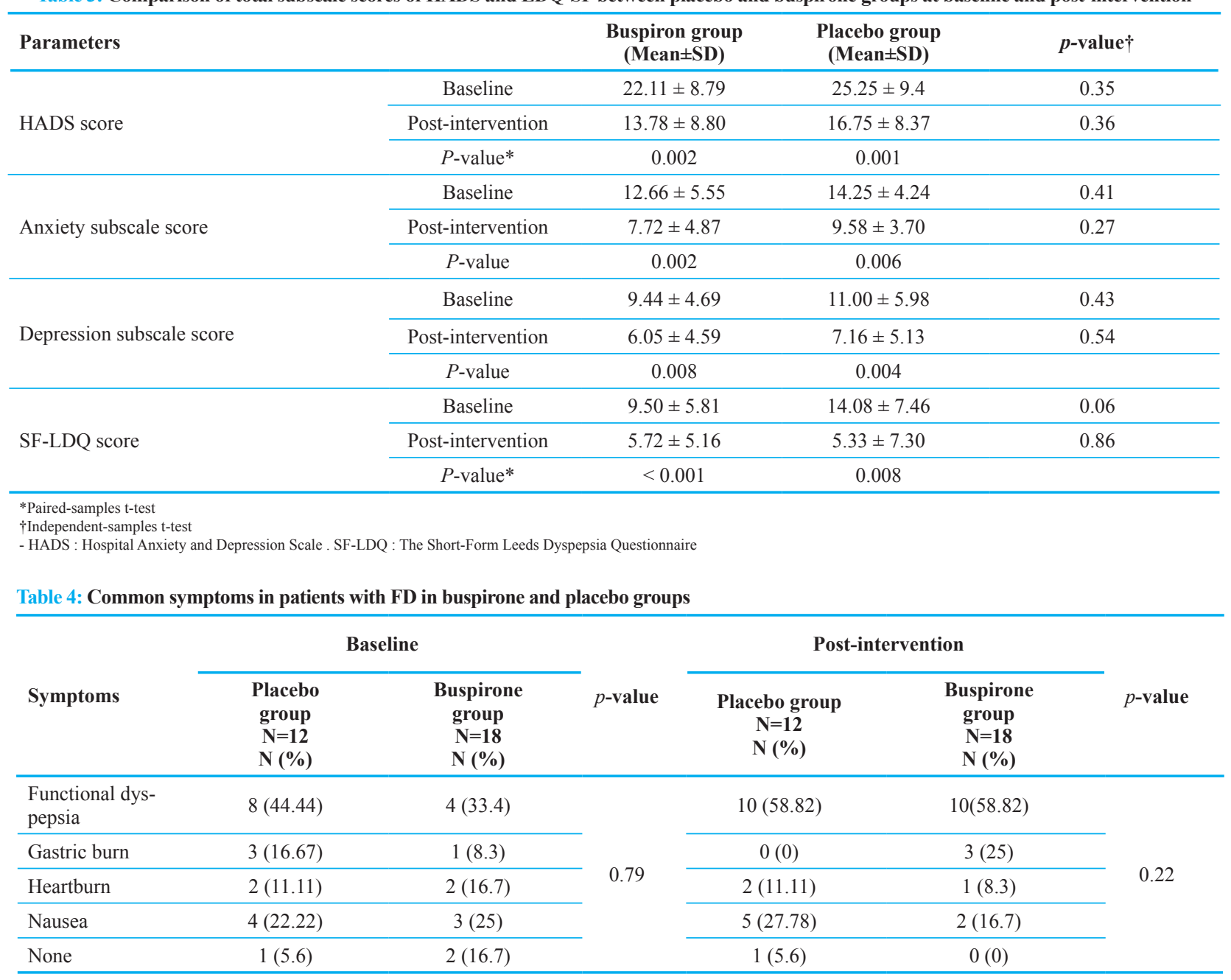

Trait Anxiety Inventory: STAI) in patients with FD. Out of the 144 patients in the mentioned study, 73 patients had received tandospirone, and 70 individuals had received a placebo. In this study, a 4-week prescription of tandospirone had significantly improved abdominal pain compared with the placebo group in weeks one, two, and four. Nevertheless, both tandospirone and placebo groups had experienced significant improvements in the QoL and psychological dimensions after intervention. ${ }^{4,9}$ A systematic review and meta-analysis in 2017 indicated that patients treated with serotonin-receptor agonists had a higher response rate in comparison with placebo-treated cases (pooled odds ratio: $\mathrm{OR}=2.99 ; 95 \%$ confidence interval: $\mathrm{CI}=1.15-7.77, p=0.05) .{ }^{19}$

Another study by Aggarwal in 2017, which was a randomized, double-blind, placebo-controlled, and crossover-based trial on patients with FD, also revealed no difference in high-resolution esophageal manometry (HREM) or the scores of symptoms in buspirone-treated patients suffering from ineffective esophageal motility/ functional dysphagia (LEM/FD) in comparison with the placebo-treated cases. ${ }^{20}$

Tabib and colleagues, in their study on Iranian patients with FD, had further compared the effects of buspirone on the QoL and clinical symptoms with those taking placebo and amitriptyline. ${ }^{8}$ The subjects had been randomly assigned to three treatment groups (i.e. $20 \mathrm{mg}$ amitriptyline, $10 \mathrm{mg}$ buspirone, and placebo) for one month. In this study, the Nepean Dyspepsia Index (NDI) had been used to evaluate the QoL and clinical symptoms before and after treatments. It had been additionally reported that buspirone was more effective than amitriptyline or 
placebo in improving the QoL, symptoms of early satiety, and abdominal pain in patients with FD. Compared with the baseline, Tabib and colleagues had described significant improvements in evaluated outcomes in all buspirone, amitriptyline, and placebo groups following the intervention, which was consistent with the findings of the present study. ${ }^{8}$

A considerable point in our study was that patients with FD receiving placebo showed improved QoL only in psychosocial dimensions. This is while both psychological and physical dimensions of QoL ameliorated in buspirone-treated patients at the end of the study. The response rate in placebo-receiving patients in clinical trials on patients with FD has also been reported to be $30-40 \%$. Nevertheless, the factors contributing to the high response rate in placebo groups in patients with FD are slightly understood. ${ }^{2}$ In fact, it is very difficult to assess the placebo effect in clinical trials or meta-analyses. This is particularly problematic as the main purpose of these studies is to reflect the outcomes of an active treatment rather than a placebo. However, potential placebo response intermediaries can be suggested as patients' expectations, classical conditioning, as well as individual beliefs. ${ }^{21}$

In 2009, Tack and others performed a placebo-controlled trial to measure the effects of R-137696, another 5-HT $\mathrm{HA}_{1 \mathrm{~A}}$ agonist, on symptoms of visceral sensitivity, gastric distension, and gastric adjustment in patients with FD. In this respect, the symptoms had been evaluated using the Patient Assessment of Gastrointestinal DisordersSymptom Severity Index (PAGI-SYM) and patients' individual symptoms subscales. In this 4-week study, 53 patients (namely, 33 with visceral sensitivity and 20 with gastric adjustment disorder) had been recruited, wherein 24 patients had received a placebo and 29 cases had taken R-137696. In line with the present study, the general symptoms scores in patients with visceral sensitivity had improved both in placebo and in R-137696-receiving groups. However, in patients with gastric adjustment disorder, none of the placebo and R-137696 groups had shown any significant improvements regarding the scores of general or individual symptoms. ${ }^{11}$

In the study by van Oudenhove and colleagues (2008) on 10 healthy volunteers (six men and four women aged 20-29 years), the effects of a single oral dose of buspi- rone $(5,10,20,30$, or $40 \mathrm{mg})$ and placebo on the tone and the sensitivity of proximal stomach were evaluated. The effects of placebo and 5, 10, and $20 \mathrm{mg}$ buspirone on gastric emptying were also assessed by a breath test of gastric emptying from solids and fluids. Compared with the pre-treatment stage, the proximal volume of the stomach increased in a dose-dependent manner with considerable fundus relaxation after 30 and $40 \mathrm{mg}$ buspirone administration. The pressure threshold did not also change during the gastric distension, but it was significantly augmented, corresponding to the volumes inside the balloon after receiving either 30 or $40 \mathrm{mg}$ buspirone. The drug significantly decelerated gastric emptying from solids and fluids and reduced gastric emptying rate at the dose of $20 \mathrm{mg}$ in healthy volunteers. ${ }^{22}$ In 2012, in a study by Tack and others, the impacts of buspirone were investigated on FD symptoms. Buspirone did not accordingly alter either the rate of gastric emptying from solids or the sensitivity to gastric distension; however, it had significantly improved gastric adjustment and delayed gastric emptying from liquids compared with the placebo. ${ }^{10}$ In another study buspirone had relaxed the proximal muscles of the stomach and had then reduced gastric emptying rate in healthy volunteers. ${ }^{19}$

The main strength of the present study was the design of the study, which was a randomized, double-blind, placebo-controlled trial.

One of the limitations of this study was the delay in reaching the maximum effects of buspirone. Data suggest that buspirone may not show its maximum effect for several weeks and may even need to reach a dose of $90 \mathrm{mg}$ per day. ${ }^{23}$ However, the duration of the present study was 8 weeks with a maximum dose of $30 \mathrm{mg}$ of buspirone. Previous studies on buspirone had been conducted for 14 days and for often 4 weeks. ${ }^{19}$ Regarding the onset of buspirone effect as a synaptic delay, there is a need to perform a study with a longer treatment period. Accordingly, the findings of the present study are expected to encourage new trials to assess the efficacy and safety of higher doses of buspirone and its longer use compared with placebos on the QoL of patients with FD. In addition, further studies are suggested to investigate significant changes in manometric parameters as well as clinical symptoms.

In conclusion, our data suggest that buspirone does 
not seem to be superior to placebo for the management of FD in Iranian patients. The changes in the QoL, anxiety, and depression as well as FD symptoms were not also significantly different between the patients receiving buspirone or placebo after 8 weeks. Further investigations are warranted to confirm these results as this study was likely underpowered to determine an effect in the context of a higher-than-expected dropout rate. Moreover, a longer follow-up period and perhaps higher doses of buspirone may demonstrate more favorable results.

\section{Authors' Contributions}

TT: Study concept and design, data collection, and statistical analysis; FE: Study concept and design, literature bibliography, development of manuscript draft, critical revision of manuscript for important intellectual content, and final revision of manuscript; ZR: data collection, literature bibliography, and statistical analysis; FN: data collection. All authors read and approved the final manuscript.

\section{ACKNOWLEDGMENTS}

We would also like to thank the patients for their cooperation in the fulfillment of this study

\section{Funding}

This study was extracted from a postgraduate thesis in Internal Medicine Specialty by Zahra Rahbar and was supported by a grant from Mazandaran University of Medical Sciences, Sari, Iran (Grant no:1397.2940) (IRCT20180405039198N1).

\section{Consent for Publication}

Not applicable.

\section{ETHICAL APPROVAL}

There is nothing to be declared.

\section{CONFLICT OF INTEREST}

The authors declare no conflict of interest related to this work.

\section{REFERENCES}

1. Zala AV, Walker MM, Talley NJ. Emerging drugs for functional dyspepsia. Expert Opin Emerg Drugs 2015;20:22133. doi: $10.1517 / 14728214.2015 .1009827$.

2. Talley NJ, Ford AC. Functional dyspepsia. $N$ Engl J Med 2015;373:1853-63. doi: 10.1056/NEJMc1515497.

3. Azizi M, Khani S, Kamali M, Elyasi F. The Efficacy and Safety of Selective Serotonin Reuptake Inhibitors and Serotonin-Norepinephrine Reuptake Inhibitors in the Treatment of Menopausal Hot Flashes: A Systematic Review of Clinical Trials. Iran J Med Sci 2021. doi: 10.30476/ ijms.2020.87687.1817.

4. Thase ME, Entsuah R, Cantillon M, Kornstein SG. Relative antidepressant efficacy of venlafaxine and SSRIs: sexage interactions. $J$ Womens Health (Larchmt) 2005;14:60916. doi: 10.1089/jwh.2005.14.609.

5. Tan VP, Cheung TK, Wong WM, Pang R, Wong BC. Treatment of functional dyspepsia with sertraline: a double-blind randomized placebo-controlled pilot study. World J Gastroenterol 2012;18:6127. doi: 10.3748/wjg.v18.i42.6127.

6. Ly HG, Carbone F, Holvoet L, Bisschops R, Caenepeel P, Arts J, et al. Mirtazapine Improves Early Satiation, Nutrient Intake, Weight Recovery and Quality of Life in Functional Dyspepsia With Weight Loss: A Double-Blind, Randomized, Placebo-Controlled Pilot Study. Gastroenterology 2013;144:S-37. doi:10.1016/S0016-5085(13)60132-8.

7. Talley NJ, Locke GR, Saito YA, Almazar AE, Bouras EP, Howden CW, et al. Effect of amitriptyline and escitalopram on functional dyspepsia: a multicenter, randomized controlled study. Gastroenterology 2015;149:340-9. e2. doi: 10.1053/j.gastro.2015.04.020.

8. Lee MJ, Choi S, Im W. 5-HT4 receptor agonists in the treatment of gastrointestinal motility disorders: current status and perspective. Int $J$ Gastroenterol Dis \& Ther 2014;1:108-116.doi: 10.15344/2393-8498/2014/108.

9. TabibSM, Nokani K, Pazoki R. The effect of Buspirone on symptoms and quality of life in patients with functional dyspepsia. Iran South Med J 2007;10:27-33.[Persian]

10. Miwa H, Nagahara A, Tominaga K, Yokoyama T, Sawada $\mathrm{Y}$, Inoue $\mathrm{K}$, et al. Efficacy of the 5-HT1A agonist tandospirone citrate in improving symptoms of patients with functional dyspepsia: a randomized controlled trial. Am J Gastroenterol 2009;104:2779 -87. doi: 10.1038/ ajg.2009.427.

11. Tack J, Janssen P, Masaoka T, Farré R, Van Oudenhove L. Efficacy of buspirone, a fundus-relaxing drug, in patients with functional dyspepsia. Clin Gastroenterol Hepatol 2012;10:1239-45 doi: 10.1016/j.cgh.2012.06.036.

12. Tack J, Van Den Elzen B, Tytgat G, Wajs E, Van Nueten L, De Ridder F, et al. A placebo-controlled trial of the 5-HT1A agonist R-137696 on symptoms, visceral hypersensitivity and on impaired accommodation in functional dyspepsia. Neurogastroenterol Motil 2009;21:619-26.e 23-4. doi: 10.1111/j.1365-2982.2008.01260.x.

13. Aziz Q, Fass R, Gyawali CP, Miwa H, Pandolfino JE, Zerbib F. Functional esophageal disorders. Gastroenterology 
2016;130:1459-65. doi: 10.1053/j.gastro.2016.02.012.

14. Sadock BJ, Sadock VA, Ruiz P. Kaplan \& Sadock's, Synopsis of psychiatry, Eleventh Edition,2016;957

15. Ware Jr JE, Sherbourne CD. The MOS 36-item shortform health survey (SF-36). I. Conceptual framework and item selection. Med Care 1992;30:473-83.

16. Montazeri Ali GA, Vahdani nia MS. Translation, Determination of Reliability and Validity of the Persian Species of the SF-36 Questionnaire. Payesh J 2005;5:49-56.

17. Kaviani H, Seyfourian H, Sharifi V,Ebrahimkhani N. Reliability and validity of Anxiety and Depression Hospital Scales (HADS): Iranian patients with anxiety and depression disorders. Tehran Univ Med J 2009;67:379-385.

18. Moayyedi P1, Duffett S, Braunholtz D, Mason S, Richards ID, Dowell AC, AT. Axon The Leeds Dyspepsia Questionnaire: a valid tool for measuring the presence and severity of dyspepsia. Aliment Pharmacol Ther 1998;12:1257-62. doi: 10.1046/j.1365-2036.1998.00404.x.

19. Jin M, Mo Y, Ye K, Chen M, Liu Y, He C. Efficacy of serotonin receptor agonists in the treatment of functional dyspepsia: a meta-analysis. Arch Med Sci 2019;15:23-32. doi:10.5114/aoms.2017.69234.

20. Aggarwal N, Thota PN, Rocio Lopez R, Gabbard S. A randomized double-blind placebo-controlled crossoverstyle trial of buspirone in functional dysphagia and ineffective esophageal motility.Neurogastroenterol Motil 2018;30: e13213.doi : 10.1111/nmo.13213.

21. Musial F, Klosterhalfen S, Enck P. Placebo responses in patients with gastrointestinal disorders. World J Gastroenterol 2007;13:3425- 3429.doi: 10.3748/wjg.v13.i25.3425.

22. Van Oudenhove L, Kindt S, Vos R, Coulie B, Tack J. Influence of buspirone on gastric sensorimotor function in man. Aliment Pharmacol Ther 2008;28:1326-33. doi:10.1111/j.1365-2036.2008.03849.x

23. Howland RH. Buspirone: back to the future. JPsychosoc Nurs Ment Health Serv.2015;53:21-24. doi: 10.3928/0279369520151022-01. 